\title{
A Comparative Study on Strengthened Community Health Services for Utilization of Malaria Control Intervention in Migori and Kwale Counties of Kenya
}

\author{
Athuman Nyae Chiguzo ${ }^{*}$, Helen Lydiah Kutima², Evan Murimi Mathenge³, \\ Charles Mwaniki Mbogo ${ }^{4}$
}

${ }^{1}$ Institute of Tropical Medicine and Infectious Diseases Jomo Kenyatta University of Agriculture and Technology. P.0 Box 62000-00200, Nairobi, Kenya. ${ }^{2}$ Jomo Kenyatta University of Agriculture and Technology (JKUAT), P.0 Box 62000-00200, Nairobi, Kenya. ${ }^{3}$ Kenya Medical Research Institute (KEMRI), P.0 Box 54840-00200, Nairobi, Kenya. ${ }^{4}$ KEMRI/Wellcome Trust Research Programme, P.0. Box 43640-00100 Nairobi, Kenya. *Corresponding Author's Email:athuman.chiguzo@gmail.com

\begin{abstract}
Malaria is the leading cause of morbidity and mortality and accounted for $40 \%$ and $30 \%$ of the outpatient attendance in 2011 in Migori and Kwale counties respectively. Perhaps, one reason for this high morbidity is the lack of capacity to deliver malaria interventions. CHVs are individuals chosen by the community and trained to address health issues of individuals, households and communities. They are catalysts, whose role is to enable individuals take control of their health. A community based comparative analytical intervention study design was implemented at baseline and four years Postintervention to measure the impact. Migori and Kwale counties were compared before and after intervention. Kwale County served as control. A baseline and follow-up Household Surveys in each of the counties were conducted to act as the pre-data and post-data respectively. In general malaria cases reduced significantly in Migori z=3.0645; 95\% CI between baseline and endline surveys compared to Kwale $\mathrm{z}=-0.8431$; 95\%CI. Testing for malaria in 2013 and 2016 in Migori increased significantly $\mathrm{z}=+27.35$; 95\%CI compared to Kwale, $\mathrm{z}=+4.799$; 95\%CI. Those who took ACTs within $24 \mathrm{hrs}$ of onset of fever increased significantly in Migori z=5.54; $95 \%$ CI compared to Kwale $\mathrm{z}=1.30 ; 95 \%$ CI. Nets in use the night before survey by children $<5$ years increased significantly in Migori $\mathrm{z}=+22.36$; $95 \% \mathrm{CI}$ compared to Kwale $\mathrm{z}=+1.04$; $95 \% \mathrm{CI}$. Nets in use the night before survey by pregnant women increased significantly in Migori $\mathrm{z}=+4.41$; $95 \% \mathrm{CI}$ compared to Kwale $\mathrm{z}=+0.679$; 95\%CI. The study concludes that involving and engaging trained CHVs contributes significantly to malaria morbidity reduction.
\end{abstract}

Key words: Malaria Morbidity, Strengthened Community Health Services, Baseline, Endline, Households.

\section{Introduction}

Malaria is the leading cause of morbidity and mortality in Kenya, accounting for $34 \%$ of the outpatient attendance in 2010 (1). Despite considerable efforts to control and treat the disease, the Kenya Demographic and Health Survey (2) estimated that about 24 million Kenyans were at risk of infection each year, with the most affected being pregnant women and children. Malaria in pregnant women contributes significantly to negative outcomes for the mother and baby, including anaemia, low birth weight and infant deaths, and it is one of the most common causes of spontaneous abortion. In addition, the ill health associated with the disease exacts a devastating toll on social and economic productivity, undermining local development in some communities (3).

Malaria is a preventable and treatable mosquitoborne disease, whose main victims are children under five years of age in Africa. The World Malaria Report 2012 summarized data received from 104 malariaendemic countries and territories for 2011.

This is an Open Access article distributed under the terms of the Creative Commons Attribution CC BY license (http://creativecommons.org/licenses/by/4.0/), which permits unrestricted reuse, distribution, and reproduction in any medium, provided the original work is properly cited.

(Received 02 August 2021; Revised 06 September 2021; Accepted 27 September 2021) 
Ninety-nine of these countries had on-going malaria transmission. According to WHO estimates, there were about 219 million cases of malaria in 2010 and an estimated 660000 deaths (4). Africa is the most affected continent; about $90 \%$ of all malaria deaths occur there. Between 2000 and 2010, malaria mortality rates fell by $26 \%$ around the world (4). In the WHO African Region the decrease was 33\%. During this period, an estimated 1.1 million malaria deaths were averted globally, primarily as a result of a scale-up of interventions (5).

Combating malaria in the long-run requires continued efforts to implement a comprehensive malaria control strategy. The Global Malaria strategy follows a fourpronged approach and includes, access to prompt diagnosis and appropriate treatment; prevention of malaria in pregnancy; vector control through the use of Long Lasting Insecticidal Nets (LLINs), Indoor Residual Spraying (IRS) and other control methods as well as a swift response to emergencies and epidemics.

Malaria is the leading cause of morbidity and mortality and accounted for $40 \%$ (6) and $30 \%$ of the outpatient attendance in 2011 in Migori and Kwale counties respectively. Perhaps, one reason for this high morbidity is the lack of capacity to deliver malaria interventions to all who need them. Community Health Strategy is the mechanism through which households and communities strengthen their role in health and health-related development issues, by increasing their knowledge and skills in disease control and planning for health interventions (7). The overall goal of the community health strategy is to enhance community access to health care in order to improve productivity and thus reduce poverty, hunger, child and maternal deaths, as well as improve educational performance (7). This strategy is driven by Community Health Volunteers (CHVs). These are individuals chosen by the community and trained to address health issues of individuals, households and communities in their localities, working in collaboration with community leaders and link healthcare facilities staff. The CHVs are catalysts, whose role is to enable individuals take control of their health, and serve as a link to the healthcare facilities when the individuals need it (7).

\section{Material and Methods}

\subsection{Study Area and Study Design}

Two counties with similar malaria endemicity, socioeconomic characteristics, geographical features, climate/weather condition and, demographic characteristics were compared before and after intervention. One county served as a control (nonintervention). The two counties are Migori county in the former Nyanza Province, (the intervention area) and Kwale county in the former Coast Province (nonintervention area). In Migori County, the study was carried out in four community health units of Nyamagagana and Gosebe in Kuria West Sub County and Itongo and Getongoroma in Kuria East Sub County. In Kwale County the study was carried out in four community health units' of Eshu and Mafisini in Msambweni Sub County and Mamba and Kikoneni in Lungalunga sub county.

A community based comparative analytical intervention study design was adopted to measure the differences in survey findings at baseline and four years post intervention follow up surveys. In each of the counties, four Community Health Units (CHUs) each with an approximate population of 5,000 people were selected. In the Intervention County (Migori), four identified CHUs were selected and established. Fourty CHVs from the established CHUs were trained on community strategy as well as malaria prevention and control interventions using Ministry of Health $(\mathrm{MOH})$ malaria Control unit approved training guidelines. A baseline and follow-up Household Survey in each of the counties were conducted to act as the pre data and post data respectively. A cross sectional study was carried out in the two counties, collecting both quantitative and qualitative data.

\subsection{Sample methodology and study population}

The study population included household members and key informant who lived within the stratified study area in both Migori and Kwale Counties. Sample size was determined using simple random sampling of households in the stratified study area in both Migori and Kwale Counties to detect significant changes in access and utilization of malaria control interventions with a $5 \%$ level of precision. The quantitative sample size was determined as described 
by Yamane, Taro (1967) (8) to evaluate the impact on community utilization of malaria control intervention in Migori and Kwale Counties, the $\mathrm{p}$ value was assumed to be 0.5 to give a sample size of 392. At baseline, the study conducted 1206 and 878 household interviews in Migori and Kwale counties respectively. At the endline survey, the study conducted 578 and 403 households' interviews in Migori and Kwale counties respectively. Eight FGDs were conducted at each of the study sites (Intervention and Control). The FGDs were conducted for groups of women, men and youths in each of the CHU giving the total of eight as stated above. In-depth Interviews (IDIs) were conducted with Sub county MOHs, Sub county malaria coordinators, opinion leaders and community health strategy coordinators making a total of eight IDIs per study site.

\subsection{Data collection tools}

Focus group and in-depth interview guides were used to generate qualitative data. Questionnaires, Rapid Diagnostic Tests (RDTs) and Artemisinin-based Combination Therapy (ACTs) consumption data tools were used to generate quantitative data. All research tools were pre tested in a local area and adjusted subsequently. Key informant interviews were also conducted to explore in-depth contextual data among specific personalities. Focus Group Discussions (FGDs) were conducted to obtain in-depth information on the subject matter.

\subsection{Data Management and Analysis}

Various quality control measures were placed to ensure the quality of information collected was guided. The measures included involvement of suitable fieldworkers and training them on the study protocol, pre-testing of study tools and supervision during data collection.

Quantitative data captured in questionnaires were coded after collection and double entered in MS Access. Any inconsistencies were dealt with immediately. To ensure consistency and completeness in qualitative data, clear requirements for the tools were continually expressed to the data collectors. At the end of each FGD and in-depth interview, there was debriefing about the salient issues arising out of the process, anomalies that occurred and what their overall opinions of the discussion/interview were. Quantitative data was entered in EPI-INFO version 7.2.1.0 and analyzed using STAT version 11.2, hypothesis tested and finally presented in tables, graphs and text. Qualitative data were sorted, aggregated from the 8 FGDs and responses of each FGD Participants, then entered by the researcher into tables broken down into themes and finally presented in tables and text. Distinctive quotes transcribed from the hand written interviews were noted in all tables and finally presented in text.

\section{Results and Discussion}

\subsection{Results}

\subsubsection{Characteristics of the study population}

In Migori, a total of 1206 and 578 households were involved in the research in the baseline and endline surveys respectively. From these households a total of 6374 and 3573 people had their information recorded for the purpose of the research during the baseline and endline surveys respectively. The average family size was 5.8 and 6 during baseline and endline surveys respectively. In Migori, gender balance was good in the research, $49.6 \%$ were males and $50.4 \%$ were females during baseline survey while $49.7 \%$ were males and 50.3 were female at endline survey. The main source of income among households was farming $85.7 \%$ at baseline survey and $77.6 \%$ at endline survey. In Kwale, a total of 878 and 430 households were involved in the research in the baseline and endline surveys respectively. From these households a total of 5529 and 2671 people had their information recorded for the purpose of the research during the baseline and endline surveys respectively. In Kwale, $49.63 \%$ were males and $50.37 \%$ were females during baseline survey while $49.81 \%$ were males and $50.19 \%$ were female at endline survey (Table. 1). 
Table 1: Socio- Economic and Demographic Profile of Study Participants in Migori and Kwale Counties

\begin{tabular}{|c|c|c|c|c|}
\hline \multirow{4}{*}{$\begin{array}{l}\text { Households visited } \\
\text { Population covered }\end{array}$} & \multicolumn{2}{|c|}{ MIGORI } & \multicolumn{2}{|c|}{ KWALE } \\
\hline & Baseline & Endline & Baseline & Endline \\
\hline & 1206 & 578 & 878 & 430 \\
\hline & 6374 & 3573 & 5529 & 2671 \\
\hline \multicolumn{5}{|l|}{ Gender } \\
\hline Male & $598(49.6 \%)$ & $285(49.3 \%)$ & $436(49.63 \%)$ & $214(49.81 \%)$ \\
\hline Female & $608(50.4 \%)$ & $293(50.3 \%)$ & $442(50.37 \%)$ & $216(50.19 \%)$ \\
\hline Average size of Household & 5.9 & 6.0 & 5.9 & 6.0 \\
\hline Minimum & 1.0 & 1.0 & 2.0 & 2.0 \\
\hline Maximum & 11.0 & 10.0 & 10.0 & 11.0 \\
\hline Average size of land (acres) & 4.5 & 6.8 & 5.0 & 7.0 \\
\hline Minimum & 0.0 & 2.0 & 3.0 & 4.0 \\
\hline Maximum & 35.0 & 24.0 & 30.5 & 50.0 \\
\hline \multicolumn{5}{|l|}{ Source of income } \\
\hline Farming & $1034(85.7 \%)$ & $449(77.68 \%)$ & $878(100 \%)$ & $430(100 \%)$ \\
\hline Self-Employment & $71(5.9 \%)$ & $47(8.20 \%)$ & $0(0 \%)$ & $0(0 \%)$ \\
\hline Formal Employment & $25(2.1 \%)$ & $17(3.0 \%)$ & $0(0 \%)$ & $0(0 \%)$ \\
\hline Business & $52(4.3 \%)$ & $57(9.9 \%)$ & $0(0 \%)$ & $0(0 \%)$ \\
\hline None & $24(2.0 \%)$ & $8(1.4 \%)$ & $0(0 \%)$ & $0(0 \%)$ \\
\hline \multicolumn{5}{|l|}{ \% HH in Possession } \\
\hline A watch & $345(28.6 \%)$ & $282(48.8 \%)$ & $187(21.6 \%)$ & $200(46.5 \%)$ \\
\hline A bicycle & $247(20.5) \%$ & $169(29.2 \%)$ & $203(23.1) \%$ & $130(30.2 \%)$ \\
\hline A motorcycle or motor scooter & $91(7.5 \%)$ & $98(17.0 \%)$ & $89(10.1 \%)$ & $65(15.10 \%)$ \\
\hline A car or truck & $72(6.0 \%)$ & $57(9.9 \%)$ & $65(7.4 \%)$ & $53(11.1 \%)$ \\
\hline A bank account & $362(20.0 \%)$ & $152(26.3 \%)$ & $255(29.0 \%)$ & $153(35.6 . \%)$ \\
\hline Mpesa Account & $611(50.66 \%)$ & $462(79.9 \%)$ & $445(50.66 \%)$ & $332(77.2 \%)$ \\
\hline Mobile Phone & $749(62.1 \%)$ & $477(82.5 \%)$ & $664(75.6 .1 \%)$ & $350(81.4 \%)$ \\
\hline
\end{tabular}

\subsubsection{Net ownership and Utilization in Migori and Kwale Counties}

Households with at least one Mosquito net (LLIN) in Migori increased from $65.8 \%$ to $92 \%$ at baseline and end line respectively while in Kwale it decreased slightly from $92.9 \%$ to $92.0 \%$ at baseline and end line respectively (table 2). Nets in use the night before survey by household members in Migori increased from $39.3 \%$ to $96.8 \%$ at baseline and endline surveys respectively while in Kwale it increased from $21.9 \%$ to $72 \%$ at baseline and endline surveys respectively. Nets in use the night before survey by children $<5$ years in Migori increased from $36.77 \%$ to $80.4 \%$ at baseline and endline respectively while in Kwale it increased slightly from $68.0 \%$ to $72.0 \%$ at baseline and endline surveys respectively.Net in use the night before Survey by pregnant women in Migori increased from $38.6 \%$ to $60.2 \%$ at baseline and endline surveys respectively while in Kwale it increased slightly from $28.5 \%$ to $36 \%$ at baseline and endline surveys respectively. The proportion of households' representative who attended a net hanging demo in Migori increased from $22.5 \%$ to 73.8 . \% at baseline and endline surveys respectively while in Kwale it increased slightly from $33 \%$ to 55\% at baseline and endline respectively. The proportion of nets hanging properly in Migori increased from $34.5 \%$ to $71.6 \%$ at baseline and endline surveys respectively while in 
Kwale it decreased slightly from $78.1 \%$ to $77.0 \%$ at baseline and endline surveys respectively (Table 2).

Table 2: Net Ownership and Utilization in Migori and Kwale Counties

\begin{tabular}{|c|c|c|c|c|}
\hline \multirow{4}{*}{$\begin{array}{l}\text { Households visited } \\
\text { Population covered }\end{array}$} & \multicolumn{2}{|l|}{ MIGORI } & \multicolumn{2}{|c|}{ KWALE } \\
\hline & Baseline & Endline & Baseline & Endline \\
\hline & 1206 & 578 & 878 & 430 \\
\hline & 6374 & 3573 & 5529 & 2671 \\
\hline Households with at least one & & & & \\
\hline Mosquito net (LLIN) & $794(65.8 \%)$ & $532(92.0 \%)$ & $816(92.9 \%)$ & $396(92.0 \%)$ \\
\hline Nets in use the night before & & & & \\
\hline $\begin{array}{l}\text { Survey by household members } \\
\text { Nets in use the night before }\end{array}$ & $2505(39.3 \%)$ & $560(96.8 \%)$ & $265(21.9 \%)$ & $310(72.0 \%)$ \\
\hline $\begin{array}{l}\text { Survey by children }<5 \text { years } \\
\text { Net in use the night before }\end{array}$ & $496(36.77 \%)$ & $608(80.4 \%)$ & $294(68.0 \%)$ & $150(72.0 \%)$ \\
\hline Survey by pregnant women & $234(38.6 \%)$ & $72(60.2 \%)$ & $16(28.5 \%)$ & $10(36.0 \%)$ \\
\hline Proportion of households & & & & \\
\hline $\begin{array}{l}\text { Representative who attend } \\
\text { a net hanging demo }\end{array}$ & $271(22.5 \%)$ & $427(73.8 \%)$ & $290(33.0 \%)$ & $237(55.0 \%)$ \\
\hline Proportion of nets hanging & & & & \\
\hline Properly & $416(34.5 \%)$ & $414(71.6 \%)$ & $686(78.1 \%)$ & $331(77.0 \%)$ \\
\hline
\end{tabular}

\subsubsection{Access to Diagnosis and Treatment of malaria Utilization in Migori and Kwale Counties}

Households with at least one Malaria case incidence decreased from $36.73 \%$ at baseline to $29.58 \%$ at endline survey in Migori compared to Kwale which decreased slightly from $63.7 \%$ at baseline survey to $61.0 \%$ at endline survey. Malaria test performed by CHVs in Migori increased significantly from $0 \%$ to $43.6 \%$ at baseline and endline surveys respectively while in Kwale increased slightly from $0 \%$ to $5 \%$ at baseline and endline surveys respectively. Fever cases that took ACT within 24 hours after its onset in Migori increased from $32.9 \%$ to $51.9 \%$ at baseline and endline surveys respectively, while in Kwale it increased slightly from $8 \%$ to $10 \%$. Positive malaria cases that received ACT from $\mathrm{CHV}$ in Migori increased significantly from $0 \%$ at baseline survey to $40 \%$ at endline survey while in Kwale it increased slightly from $0 \%$ to $5 \%$ at baseline and endline surveys respectively (Table 3). 
Table 3: Access to Diagnosis and Treatment of malaria Utilization in Migori and Kwale Counties

\begin{tabular}{|c|c|c|c|c|}
\hline \multirow{4}{*}{$\begin{array}{l}\text { Households visited } \\
\text { Population covered }\end{array}$} & \multicolumn{2}{|l|}{ MIGORI } & \multicolumn{2}{|l|}{ KWALE } \\
\hline & Baseline & Endline & Baseline & Endline \\
\hline & 1206 & 578 & 878 & 430 \\
\hline & 6374 & 3573 & 5529 & 2671 \\
\hline \multicolumn{5}{|l|}{ Households with at least one } \\
\hline Malaria case incidence & $443(36.73 \%)$ & $171(29.58 \%)$ & $560(63.7 \%)$ & $262(61.0 \%)$ \\
\hline Number tested for malaria & $546(45.5 \%)$ & $540(93.5 \%)$ & $351(40.0 \%)$ & $232(54.0 \%)$ \\
\hline Tests performed by CHVs & $0(0.0 \%)$ & $252(43.6 \%)$ & $0(0.0 \%)$ & $23(5.4 \%)$ \\
\hline Malaria tests results positive & $1105(96.6 \%)$ & $494(85.4 \%)$ & $717(81.6 \%)$ & $206(48.0 \%)$ \\
\hline Malaria tests results negative & $101(8.4 \%)$ & $84(14.6 \%)$ & $162(18.4 \%)$ & $206(48.0 \%)$ \\
\hline \multicolumn{5}{|l|}{ How Long after fever started was } \\
\hline ACT taken within 24 hours & $397(32.9 \%)$ & $300(51.9 \%)$ & $70(8 \%)$ & $43(10.0 \%)$ \\
\hline Positive cases that Received ACT & $1172(97.2 \%)$ & $574(99.3 \%)$ & $878(100 \%)$ & $430(100 \%)$ \\
\hline \multicolumn{5}{|l|}{ Those that Received ACT from } \\
\hline Public health facilities & $548(45.4 \%)$ & $293(50.69 \%)$ & $685(78.0 \%)$ & $344(80.0 \%)$ \\
\hline Those who received ACT from CHV & $0(0.0 \%)$ & $231(40.0 \%)$ & $0(0 / 0 \%)$ & $22(5 \%)$ \\
\hline
\end{tabular}

\subsubsection{Health Facility Confirmed Malaria Cases (Data Linked to Study area CHUs in Migori County [Intervention])}

The mean annual malaria cases above five years for all facilities decreased from 3074 in 2013 to 1537 in $2016, \mathrm{z}=2.1104$; 95\%CI. The mean annual malaria cases under five years for all facilities decreased significantly from 2628 in 2013 to 883 in 2016, $\mathrm{z}=2.9371$; 95\%CI (Figure 1).

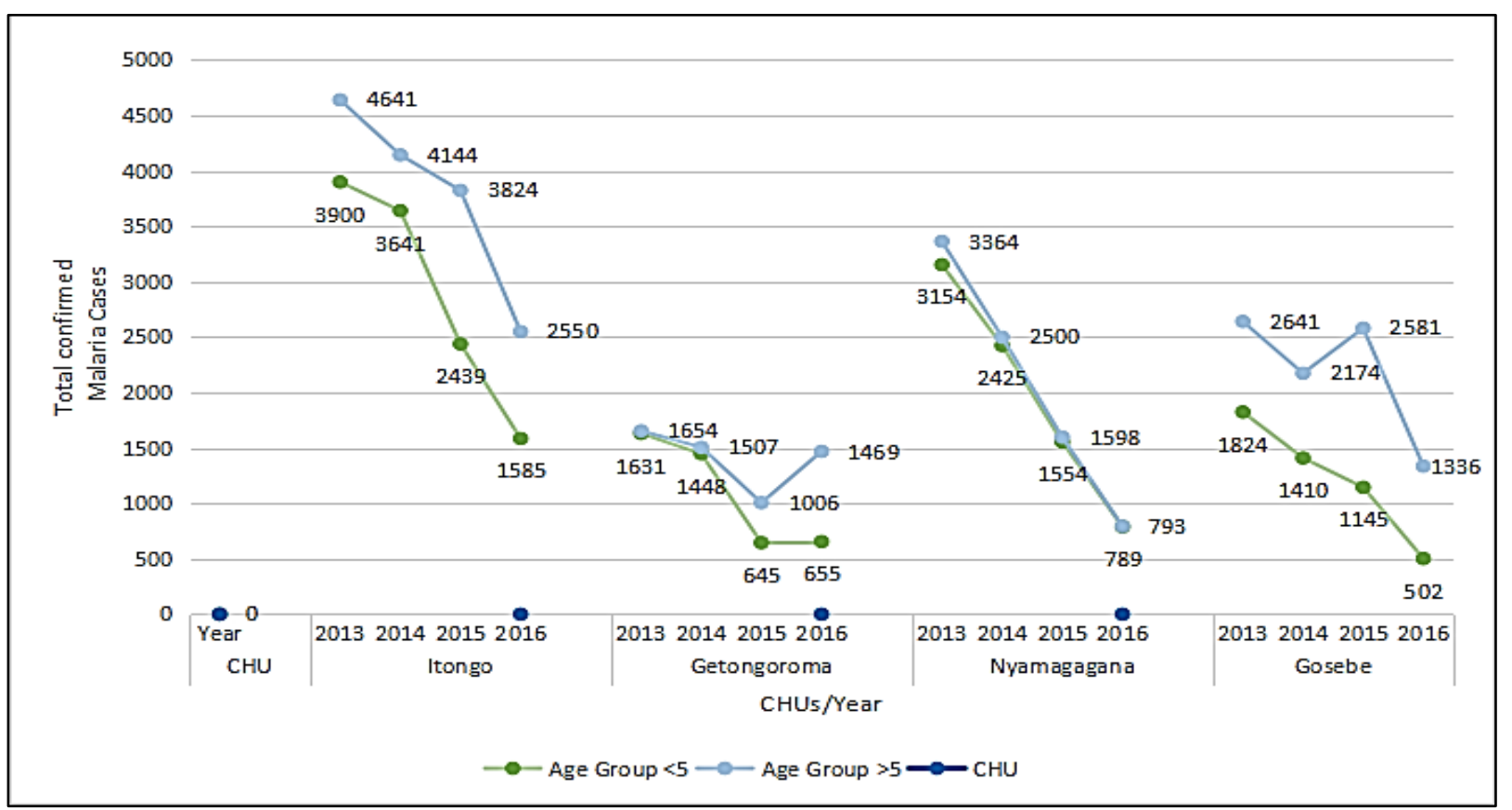

Figure 1: Confirmed malaria cases Migori County CHUs 2013 to 2016 


\subsubsection{Health Facility Confirmed Malaria Cases (Data Linked to Study area CHUs in Kwale County [None Intervention]}

The mean annual malaria cases for above five years for all facilities decreased from 2261 in 2013 to 1512 in 2016, z=-0.90175; 95\%CI. The mean annual malaria cases for under five years for all facilities increased from 1068 in 2013 to 1515 in 2016, z =05247; 95\%CI (Figure 2).

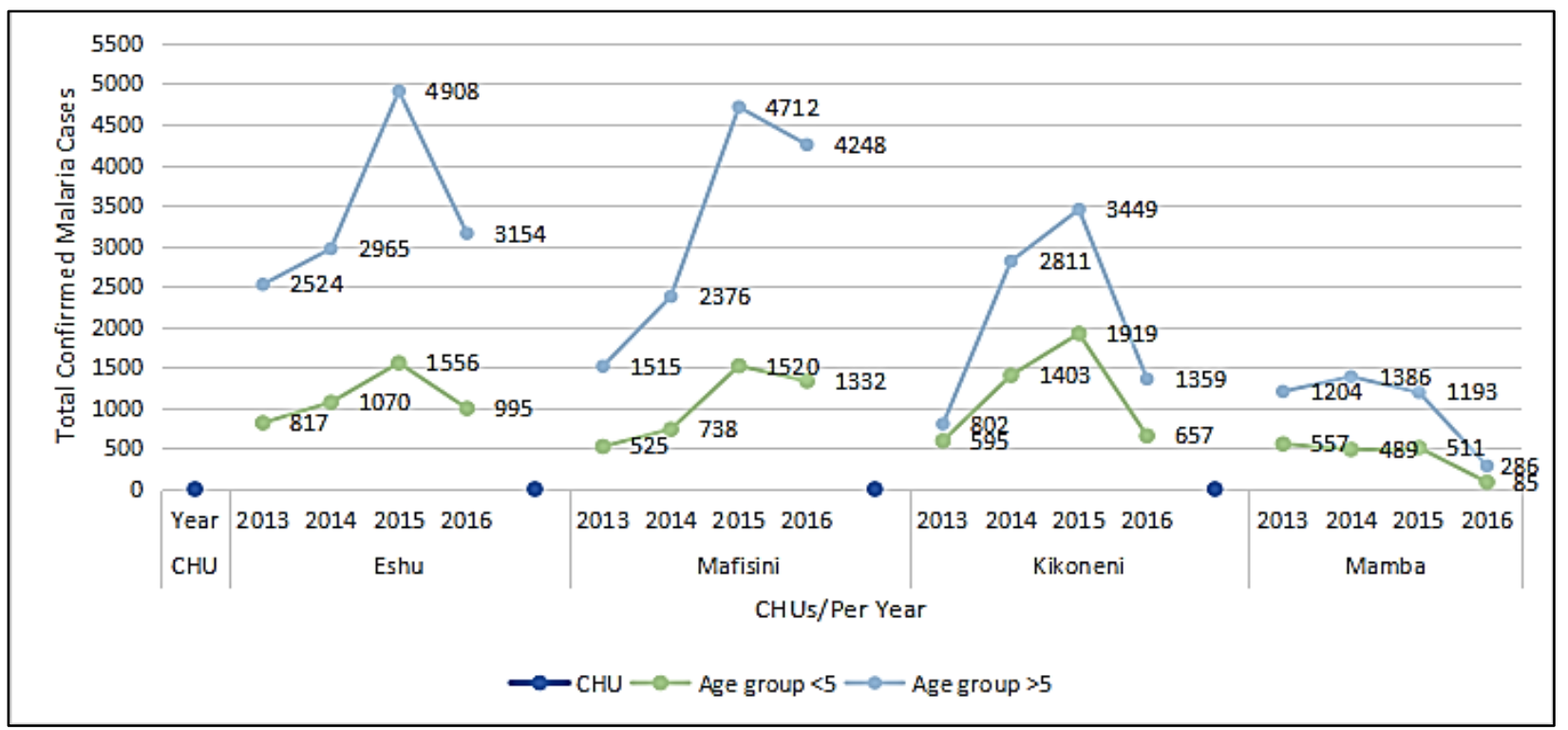

Figure 2: Confirmed malaria cases Kwale County CHUs 2013 to 2016

3.1.6 Health Facility Confirmed Malaria Cases (Data Linked to Study area CHUs in Migori County [Intervention] and Kwale County [None. Intervention])

The mean annual malaria cases above five years for all facilities decreased significantly from 3075 in 2013 to 1537 in 2016 in Migori County, z=2.1104; 95\%CI compared to Kwale County decreased from 2261 in 2013 to 1512 in 2016, z =-0.90175; 95\%CI. The mean annual malaria cases under five years for all facilities decreased

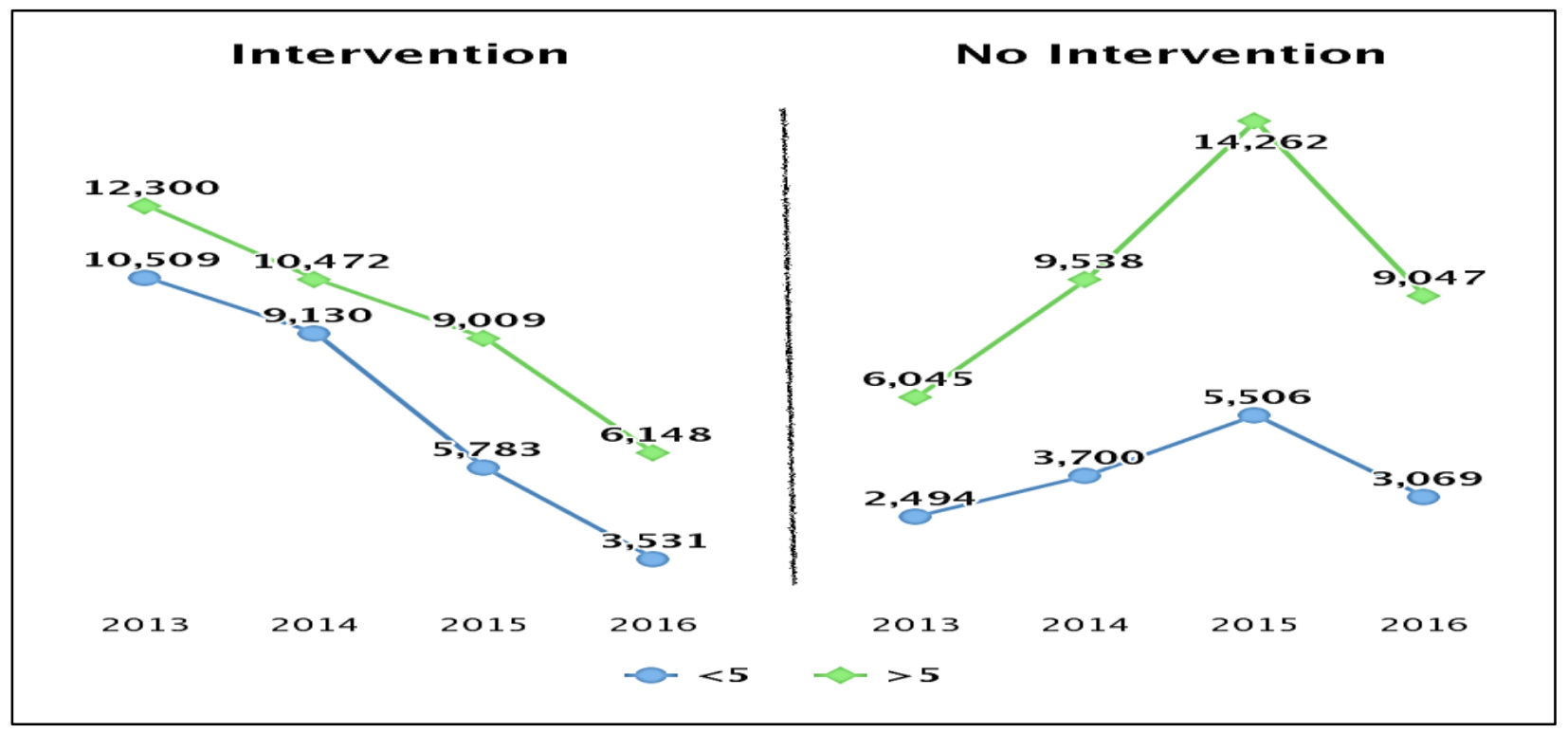

Figure 3: Total Confirmed malaria cases in Migori and Kwale County CHUs 2013 to 2016 
Table 4: Access and Utilization of Malaria Prevention in Pregnancy in Migori and Kwale Counties

\begin{tabular}{|c|c|c|c|c|}
\hline \multirow[b]{3}{*}{ Households visited } & \multicolumn{2}{|l|}{ MIGORI } & \multicolumn{2}{|c|}{ KWALE } \\
\hline & Baseline & Endline & Baseline & Endline \\
\hline & 1206 & 578 & 878 & 430 \\
\hline Population covered & 6374 & 3573 & 5529 & 2671 \\
\hline Pregnant Women & 115 & 37 & 56 & 27 \\
\hline \multicolumn{5}{|c|}{ Pregnant women currently } \\
\hline Attending ANC & $69(60.0 \%)$ & $34(91.9 \%)$ & $18(31.4 \%)$ & $15(54.5 \%)$ \\
\hline \multicolumn{5}{|c|}{ ANC attending women who have } \\
\hline Received IPTp2 & $20(29.10 \%)$ & $16(47.1 \%)$ & $6(31.4 \%)$ & $8(54.5 \%)$ \\
\hline \multicolumn{5}{|c|}{ ANC attending women who have } \\
\hline Received IPTp3 & $14(20 \%)$ & $12(35.3 \%)$ & $3(18.2 \%)$ & $0(0 \%)$ \\
\hline
\end{tabular}

significantly from 2628 in 2013 to 883 in 2016 in Migori County, $z=2.9371$; 95\%CI compared to Kwale County that increased from 1068 in 2013 to 1515 in 2016, z=-0,5247; 95\%CI. In general malaria cases reduced significantly in Migori $\mathrm{z}=3.0645$ between baseline and endline surveys compared to Kwale $\mathrm{z}=$ 0.8431; 95\% CI over the same period (Figure 3).

\subsubsection{Access and Utilization of Malaria Prevention in Pregnancy in Migori and Kwale Counties}

Pregnant women currently attending ANC in Migori increased from $60 \%$ to $91.9 \%$ at baseline and endline surveys respectively while in Kwale it increased from $31.4 \%$ to $54.4 \%$ in the same period. ANC attending women who had received IPTp3 in Migori increased from $20 \%$ to $35.3 \%$ at baseline and endline respectively while in Kwale it decreased from 18.2\% to $0 \%$ ) over the same period (Table 4 ).

A few of the respondents (21\%) in Migori mentioned that they had a woman of child bearing age in their household who was pregnant during FGD at baseline survey. Only $11.9 \%$ received medicine for malaria prevention using Intermittent Presumptive Treatment in Pregnancy with Sulphurdoxine Pyramethamine (IPTpSP). Majority (89\%) of the respondents during FGD mentioned they were not aware about malaria prevention using IPTp. Majority of the respondents (80\%) did not know how many times a pregnant woman must take medicine for malaria prevention (IPTp) at baseline survey in Migori.

"I am not aware that pregnant women get malaria medicine for prevention. I even do not how many times they are supposed to take". A woman respondent from Glory women group in Kuria East Sub County during FGD at the baseline survey. However, during FGD at endline survey in Migori, 25\% of the respondents mentioned they had a woman of child bearing age in the households who was pregnant. All respondents during FGD at endline survey mentioned that they were aware of malaria prevention using IPTp. We are all aware that pregnant should take IPTp. This is because every time the CHV visits our households they remind every pregnant woman to attend ANC so that they get IPTp". Women group respondents from Masaba in Kuria west Sub County during FGD at endline survey.

\subsection{Discussion}

Insecticide-treated bed nets (ITNs) form a protective barrier around persons using them. They have been shown to reduce severe disease and mortality due to malaria in endemic regions. Several studies have also demonstrated the efficacy of ITNs with an overall reduction in all-cause mortality by 19\% [9]. A $25 \%$ 
reduction in all-cause mortality for children one to nine years of age was detected during the first year of the Gambian National Bed net Program [10]. In Kilifi District, Kenya, a 33\% reduction in mortality and a $44 \%$ reduction in hospital admissions for severe malaria were also found [11].

Long Lasting Insecticide Treated Nets (LLINs) are one of today's most effective malaria prevention interventions. They have been shown to reduce severe disease and mortality due to malaria in endemic regions among the vulnerable groups (pregnant women, children $<5$ ). They are mainly recommended for pregnant women, children $<5$ and HIV positive people. LLIN use is encouraged early and consistently throughout pregnancy and after delivery [12]. The LLIN retain lethal concentrations of insecticide for at least 3 years. This study found out that the proportion of households owning at least one net increased significantly from $65.8 \%$ in 2013 to $92 \%$ in 2016 in Migori County compared to Kwale County that decreased slightly from $92.9 \%$ in 2013 to $92.0 \%$ in 2016. The proportion of net use the night before the survey by children $<5$ increased from $36.8 \%$ to $80.4 \%$ and by pregnant women increased from $36.6 \%$ to 60.2\% in 2013 and 2016 in Migori $(\mathrm{z}=22.36$ and 4.41; $95 \%$ CI respectively) compared to Kwale County by children $<5$ increased slightly from $68.0 \%$ to $72.0 \%$ and by pregnant women from $28.5 \%$ to $36.0 \%$ ( $\mathrm{z}=1.04$ and $0.679 ; 95 \%$ CI respectively) over the same period. These findings compares favourable with those of LLIN ownership in the 2010 and in the 2015 KMIS surveys [13]. Nationally, ownership of at least one LLIN increased by 19 percentage points since 2010, from 44 percent to 63 percent. It is important to note that Migori County received two rounds of free LLINs distribution in 2014 and 2016 while Kwale County had received only one round of free LLINs distribution in early 2015 [14]. These findings are consistent with global figures. Globally, across sub-Saharan Africa, household ownership of at least one ITN increased from $50 \%$ in 2010 to $80 \%$ in 2016 [15]. However, the proportion of households with sufficient nets (i.e. one net for every two people) remains inadequate, at $43 \%$ in 2016 [15]. In this study, the proportion of households' representative who attended a net hanging demo in Migori increased from $22.5 \%$ to $73.8 \%$ at baseline and endline surveys respectively while in Kwale it increased slightly from $33 \%$ to $55 \%$ at baseline and endline surveys respectively. At the same time, the proportion of nets hanging properly in Migori increased from 34.5\% to $71.6 \%$ at baseline and endline surveys respectively while in Kwale it decreased slightly from 78.1\% to $77.0 \%$ at baseline and endline surveys respectively. Nets in use the night before the Survey by pregnant women in Migori increased significantly from 38.6\% to $60.2 \%(\mathrm{z}=4.41 ; 95 \% \mathrm{CI})$ at baseline and endline surveys respectively while in Kwale it increased slightly from $28.5 \%$ to $36 \% \quad(\mathrm{z}=1.04 ; 95 \% \mathrm{CI})$ at baseline and endline surveys respectively. These findings suggests that CHVs in Migori had a greater role of motivating and promoting the use of nets among household members, children $<5$ and pregnant women compared to their counterparts in Kwale. CHVs in half of the CHUs (Kikoneni and Mamba) in Kwale received just one training on integrated Malaria, HIV and TB management and prevention as well as community health strategy. The other half of the CHUs (Mafisini and Eshu) did not receive any form of training. Perhaps the regular training, motivation and support of the CHV in Migori contributed to this outcome.

CHVs who are the main drivers of the community health services (foot soldiers) received three trainings in Migori County during the study period. One training on the community health strategy module for CHVs, training on community malaria case management training module and a refresher training on the same in year 3 of the intervention study. They received a monthly stipend of Ksh. 2,000/= throughout the study period. They received a bicycle each, a drug-kit which they kept replenishing at the link-health facility as well as got uniforms and badges. They participated in most of the community health activities such health promotion, net distributions, net hanging campaigns, national immunization services (measles and polio), growth monitoring, family planning and other health promotion activities. The Ministry of Health through the National Malaria Control Program's Strategy for universal free net distribution which undertakes a campaign every three years has not only increased net ownership in households but also increased net utilization by 
household members. Routine net distribution for children $<5$ and pregnant women is done throughout the year and nets distributed through ANC and Child Health Welfare Clinic's. During the four year period of the study, free net distribution took place twice in Migori County in September 2014 under FY 2014/15 and in November 2016 under FY 2016/17 compared to Kwale that took place only once in August 2015 under FY 2014/2015. Trained CHVs in Migori were pivotal in LLINs distribution campaigns at the household as well as in the actual net distribution for universal coverage. Perhaps this activity increased their recognition, motivation, visibility, as well as acceptances at the household level in their area of jurisdiction to promote demand and use of malaria control interventions and health promotion.

The ultimate goal of malaria control is to reduce morbidity and prevent mortality due to malaria thereby mitigating the socio-economic burden of the diseases in Kenya. One of the key strategic interventions is to provide early parasitological diagnosis and prompt treatment using effective medicine (16). ACTs are at present the best treatment for uncomplicated malaria and the efficacy of the treatment recommended in the current guidelines for the diagnosis, treatment and prevention of malaria in Kenya (17). In this study, malaria morbidity among the general population in Migori County reduced statistically significant from $36.7 \%$ in 2013 to $29.6 \%$ in 2016 ( $\mathrm{z}=2.175 ; 95 \% \mathrm{CI}$ ) compared to Kwale where it decreased slightly from $63.7 \% \%$ to $61.0 \%(\mathrm{z}=0.679 ; 95 \% \mathrm{CI})$. These findings are further supported by FGDs mentioning that malaria incidence decreased in households in Migori from $97.36 \%$ to $25 \%$ at baseline and endline surveys respectively. While in Kwale this increased from $70.3 \%$ to 91 . 9\% at baseline and endline surveys respectively. In addition, while further comparing the mean annual malaria morbidity among children under five in all health facilities in Migori a statistically significant decrease from 2628 cases in 2013 to 883 in 2016 was noted, $\mathrm{z}=2.9371$; $95 \% \mathrm{CI}$, whereas in Kwale there was an increase from 1068 cases in 2013 to 1515 in 2016, $\mathrm{z}=-0.5247 ; 95 \% \mathrm{CI}$. This suggests a greater reduction in malaria morbidity among children under five in Migori compared to Kwale. These findings are supported by the FGDs where majority (90\%) of the respondents from both focus group discussions and key informant interview in Migori County mentioned that they had observed a reduction in malaria morbidity among the general population and children $<5$ in 2016 endline survey compared to 2012 baseline survey. While in Kwale County over the same period, majority of the respondents' (85\%) of FGDs and key informant interviews mentioned they had observed a slight increase in malaria morbidity in 2016 compared to 2013.

These findings are further supported by FGD findings whereby participants mentioned that people who took ACTs within $24 \mathrm{hrs}$ of developing a fever increased from $53.8 \%$ to $72.3 \%$ at baseline and endline surveys respectively in Migori, while in Kwale there was no difference between baseline and endline surveys (30\%).These findings are consistent with the findings of the Kenya malaria indicator surveys conducted in 2010 and 2015 showing a reduction in morbidity from $38 \%$ to $27 \%$ in the lake endemic region while coast endemic region showed a slight reduction from $11 \%$ to $8 \%$ over the same period. These findings are consistent with the world malaria report 2019, where the incidence rate of malaria declined globally between 2010 and 2018, from 71 to 57 cases per 1000 population at risk. However, from 2014 to 2018, the rate of change slowed dramatically, reducing to 57 in 2014 and remaining at similar levels through to 2018 (18). In 2020, WHO reported that the global tally for malaria in 2019 was 229 million cases and had remained virtually unchanged over the past four years. Malaria claimed 409,000 lives in 2019 compared to 411,000 in 2018 (19). According to a report from the department of health services in Kwale County, malaria had increased from a prevalence of $8 \%$ in 2015 to $12 \%$ in 2017 (20). In this study, these findings suggests that establishing community health units and making them operational has positive health impact especially on reduction of malaria morbidity among the general population and even greater among children under five. Perhaps the main reason is access and utilization of malaria control interventions triggered by the activities of CHVs during regular household visits. These findings are consistent with H Perry \& P Freeman, 2009 
(21)where they found increasing evidence demonstrating that community and women's empowerment can have a remarkable impact on the health of children. Karen LeBan et al., (22) also demonstrated that high-impact health prevention and promotion activities, such as exclusive breastfeeding; infant and young child feeding; birth delivery planning; use of an insecticide-treated net for malaria prevention; essential newborn care can be realized in a community setting. This is particularly of high importance in community settings where behavior change approaches are essential, than in facility settings where more complex medical procedures are promoted for maximum impact. Suffice to say in this study is that the CHVs activities have demonstrated behaviour change at households on utilization of malaria control interventions. For instance, ACTs taken within 24 hours after onset of fever increased significantly from $32.9 \%$ to $51.9 \%$ at baseline and endline surveys respectively in Migori, while in Kwale a slight increase from $8.0 \%$ to $10.0 \%$ over the same period. These findings are further supported by FGD findings where by participants mentioned fever cases that took ACT within 24 hours after its onset in Migori increased from $53.8 \%$ to $72.3 \%$ at baseline and endline surveys respectively.

On recorded confirmed malaria cases (Figure 2) shows a reduction between 2015 and 2016 in Kwale County with a greater reduction being exhibited in Mamba and Kikoneni CHUs. This is because in 2014, Kwale County adopted the community health strategy. Community health units were formed in Mafisini and Eshu in Msambweni Sub County as well as Mamba and Kikoneni in Lungalunga Sub County. At the beginning of 2015 free nets were distributed in Kwale County. In 2015 through the support of Global fund, WOFAK (Women Fighting Aids in Kenya) established $40 \mathrm{CHU}$ in the entire Lungalunga sub county as well as trained all the 401 CHVs on community malaria case management. They also gave 500 bicycles, one for each CHV and at least 2 for each CHU.

These observations suggest that strengthening level one health system can impact on health outcomes and conforms to the findings of Karen LeBan et al., (22).
Malaria infection during pregnancy is a major public health problem, with substantial risks to the mother, her foetus and the newborn. During pregnancy there is altered/ suppressed immune response to malaria especially in first and second gravidae as well as HIV infected persons. Malaria infection in pregnancy can cause maternal complications, morbidity including anaemia and mortality.

The goal of prevention of malaria in pregnancy is to reduce maternal and perinatal morbidity and mortality associated with malaria. The strategies in prevention of malaria in pregnancy are integrated in the overall antenatal care (ANC) package for maternal health. To prevent the irreversible, negative consequences of malaria during pregnancy, it is critical to encourage pregnant women to sleep under LLINs and start IPTp-SP regimen as early as possible in the second trimester. WHO and partners recommend initiating malaria prevention efforts beginning at 13 weeks to achieve major benefits. The period between 13 and 20 weeks is critical because this is the time when the placenta is forming and the parasite densities are highest. IPTp is an antimalarial given in treatment doses at defined intervals. It assumes every woman in a malaria endemic area has malaria parasites in blood or placenta. IPTp prevents placental malaria infection, low birth weight associated with early infant mortality.

The study found a high increase in pregnant women who attended ANC in Migori from 60\% to $91.9 \%$ $(\mathrm{z}=4.94 ; 95 \% \mathrm{CI})$ at baseline and endline respectively while in Kwale it increased moderately from $31.4 \%$ to $54.4 \%$ ( $\mathrm{z}=2.02 ; 95 \% \mathrm{CI}$ ) over the same period. Thus, pregnant women in Migori are more than twice likely to attend ANC compared to those of Kwale. Nationally, ANC services are universally promoted in the country. The disparity between Migori and Kwale in terms of utilization of ANC services by pregnant women over the same period is perhaps because of the pregnant women motivations by trained CHVs in the former than the later. Respondents from Focus Group Discussions in Migori mentioned that CHVs promoted the use of IPTp which is administered at ANC clinics during the endline survey. However, in Kwale CHVs 
promoted the use of IPTp mainly in Mamba and Kikoneni CHUs that were established by WOFAK (23). The study found out that ANC attending women who had received IPTp3 in Migori increased from 20\% to $35.3 \%$ ( $\mathrm{z}$ value $=+1.321 ; 95 \% \mathrm{CI}$ ) at baseline and endline respectively compared to Kwale that decreased significantly from $18.2 \%$ to $0 \%$ ( $\mathrm{z}$ value $=$ 3.53; 95\% CI).

These findings are consistent with those of KMIS of 2010 and 2015. In Kenya, there has-been increased IPTp uptake over the years but has not reached the national target of $80 \%$ [17]. IPTp1: $56-79 \%$ between 2015 and 2017.Analysis of data reported by country programmes and data available through household surveys shows relatively high levels of ANC attendance (88\%, IQR 68\%-95\%) but much lower proportions of women attending ANC receiving IPTp3 (44\%, IQR 30\%-57\%). These findings are further supported by the FGDs during baseline and endline surveys in Migori. Thus, a few of the respondents (21\%) mentioned that they had a woman of child bearing age in their household who was pregnant during FGD at baseline survey. Only 11.9\% received medicine for malaria prevention (IPTp). Majority $(80 \%)$ of the respondents did not know how many times a pregnant woman must take IPTp. A woman respondent from Glory women group in Kuria East Sub County during the baseline survey said, "I am not aware that pregnant women get malaria medicine for prevention. I even do not how many times they are supposed to take".

Thus at baseline survey, the study found that the awareness of the community about malaria prevention using IPTp was very low. Majority (89\%) of the respondents during FGDs at baseline survey mentioned they were not aware about malaria prevention using IPTp. However, at endline survey because of the activities of health promotion by the CHVs in the Intervention area, awareness on the use of IPTp at the household level increased. All respondents during FGD at endline survey mentioned they were aware about malaria prevention using IPTp. "We are all aware that pregnant women should take IPTp. This is because every time the CHV visits our households they remind every pregnant woman to attend ANC so that they get IPTp." Women group respondents from Masaba in Kuria west Sub County during FGD at endline survey.

The overall ANC package for maternal health in Kenya provides access to IPTp using SP in all health facilities that provide ANC services. All respondents during FGDs mentioned that SP was available at the ANC clinics whenever they attended.

"When a pregnant woman visits an ANC clinic they always receive IPTp-SP".A woman respondent from Glory women group in Kuria East Sub County during the endline survey FGDs. Thus, access may not be the real issue but probably utilization is inhibited by other factors such as contraindications for SP which is Sulphur based medicine or other cultural beliefs and practices that were not explored by this study. Perhaps the alternative of prevention of malaria in pregnancy using LLINs was more preferred to IPTp. Thus the study established that use of LLINs by pregnant women on the night before the survey in Migori improve significantly from $38.26 \%$ to $60.2 \%$ at baseline and endline surveys respectively $(\mathrm{z}=4.94$; 95\% $\mathrm{CI})$.

\subsection{Conclusion}

In conclusion, the study has demonstrated that, strengthening community health services at (level 1) leads to great improvement in community utilization of malaria control interventions such as Net ownership and utilization, Access and utilization of malaria prevention in pregnancy (IPTp3) as well as Access to diagnosis and treatment utilization of malaria, This has a great effect in reducing malaria morbidity and mortality as desired in the Kenya's National Malaria Control Strategy. Further research should be conducted on the economic evaluation of the community health services as well as cost benefit analysis on the remuneration of community health volunteers.

\section{Acknowledgements}

We wish to convey our gratitude to the research teams both in Kwale and Migori Counties that participated in data collection. Thanks to the Director KeNAAM who was able to link me with his Global 
Fund Malaria project team in Migori. Special thanks to all the participants who took part in the study. Special thanks go to my supervisors who have been of great encouragement to me and have constructively critiqued this study to make it better. I would also like to appreciate the timely support and encouragement from the management of Jomo Kenyatta University of Agriculture and Technology (JKUAT), and KEMRI. We would like to acknowledge the Director KEMRI.

\section{Conflict of Interest}

The authors declare that there is no conflict of interests regarding the study or this article.

\section{Funding Sources}

The funding of this study was privately done by the researchers.

\section{Ethics}

The study proposal was submitted to the Maseno University Ethical Review Committee (MUERC) for review and approval. Prior to the start of the study, permission was sought from the local community leadership and other relevant authorities in the selected areas in which the study was conducted.

\section{References}

1. Kenya National Bureau of Statistics and ICF M ACRO (2011). Kenya Malaria Indicator Survey (KNMS), K.N.B.o.S.a.I. Macro, Editor. 2011: Nairobi. Control, DOMC, National Malaria Strategy 2009-2017. 2009: NAIROBI.

2. Kenya National Bureau of Statistics and ICF M ACRO (2010). 2008-2009 Kenya Demographic Health Survey. Kenya National Bureau of Statistics and ICF Macro (2010). 2008-2009 Kenya Demographic Health Survey.

3. Kenya National Bureau of Statistics (2012).Division of Malaria Control [Ministry of Public Health and Sanitation], Kenya National Bureau of Statistics, Population Services International Kenya, and ICF Macro. 2012. 2011/12 Evaluation of the Post Mass Net Distribution Programme. Nairobi, Survey, Evaluation Kenya Malaria Indicator Survey. Nairobi, Kenya: DOMC, KNBS, PSI, and ICF Macro.

4. World Health Organization (2012). World malaria report 2011. Geneva, World Health Organization, 2011, (http://www.who.int/entity/malaria/world_malaria_repor t 2011/9789241564403_eng.pdf, accessed 10 November 2012).

5. World Health Organization (2012). Global plan for insecticide resistance management in malaria vectors (GPIRM). Geneva: World Health Organization, 2012.

6. Migori County Malaria Profile 2012.
7. Ministry of health, (2006). Taking the Kenya Essential Package for Health to the community. A strategy for Delivering of Level One Services, Nairobi Kenya, 2006.

8. Yamane, Taro (1967) Statistics. An introductory analysis, 2nd Edition, New York, Harper and Row.

9. Phillips-Howard PA, Nahlen BL, Kolczak MS, Hightower AW, Kuile FO, Alaii JA, Gimnig JE, Arudo J, Vulule JM, Odhacha A, Kachur SP, Schoute E, Rosen DH, Sexton JD, Oloo AJ, Hawley WA. Efficacy of pyremethrin-treated bed nets in the prevention of mortality in young children in an area of high perennial malaria transmission in western Kenya. Amer J Trop Med Hyg. 2003; 68 (4): 23-29.

10. D'Alessandro U, Olaleye BO, McGuire W, Thomson MC, Langerock P, Bennett S, Greenwood BM.. A comparison of the efficacy of insecticide-treated and untreated bed nets in preventing malaria in Gambian children. Trans. R. Soc. Med. Hyg. 1995; 89 (6): 596-8.

11. Nevill C, Some E, Mung'ala V, Mutemi W, New L, Marsh $\mathrm{K}$, Lengele, C, Snow R. Insecticide-treated bed nets reduce mortality and severe morbidity from severe malaria among children on the Kenyan coast. Trop Med Int. Health. 1996; 1:139-146.

12. Yartey JE. Malaria in pregnancy: access to effective interventions in Africa. Int. J Gynaecol Obstet. 2006; 94(3):364-73.

13. Kenya Malaria Indicator Survey 2015 (NMCP), K.N.B.o.S.a.I. Macro, Editor. 2016: Nairobi

14. Ministry of Health, 2015. Ministry of Health Kenya circular on free nets distribution.

15. World Health Organization, 2017. World Malaria Report 2017. Geneva: World Health Organization; 2017. Licence: CC BY-NC-SA 3.0 IGO.

16. Ministry of Health, 2018. Guidelines for the diagnosis, treatment and prevention of malaria in Kenya, Fifth Edition revised 2018.

17. Ministry of Health 2016. Trainers Manual for diagnosis, management and prevention of malaria, Republic of Kenya, 2016. .

18. World Health Organization. World malaria report 2019. Geneva: World Health Organization; 2019. Licence: CC BYNC-SA 3.0 IGO.

19. World Health Organization: World Malaria report 2020: 20 years of global progress and challenges Geneva. World Health Organization; 2020 Licence: CC BY-NC-SA 3.0 IGO 20. Kwale County Department of Health Services (2018). Kwale County Consolidated Annual Work Plan 2018-2019. Department of Health Services, 2018.

21. H Perry and P. Freeman. How effective is communitybased primary health care in improving the health of children? Summary findings. Report to the Expert Review Panel. A review of the evidence. 2009.

22. Karen LeBan et al., 2011 (CORE Group). Understanding community components of a Health System draft for input: May 1, 2011.

23. Ministry of Health 2018. Rapid Assessment report on the Implementation of Integrated HIV, TB and Malaria Services at the Community Level in Rangwe, Emuhaya and Lungalunga Sub-Counties, Kenya. The Community System Strengthening Technical Working Group (TWG) September 1, 2018. 